\title{
CONCAVE RENEWAL FUNCTIONS DO NOT IMPLY DFR INTERRENEWAL TIMES
}

\author{
YAMING YU, ${ }^{*}$ University of California
}

\begin{abstract}
Brown (1980), (1981) proved that the renewal function is concave if the interrenewal distribution is DFR (decreasing failure rate), and conjectured the converse. This note settles Brown's conjecture with a class of counterexamples. We also give a short proof of Shanthikumar's (1988) result that the DFR property is closed under geometric compounding.
\end{abstract}

Keywords: Renewal theory; log-convexity

2010 Mathematics Subject Classification: Primary 60K05

\section{Introduction}

Structural relationships between the renewal function and the underlying distribution are of great interest in renewal theory. For a renewal process with decreasing failure rate (DFR) interrenewal times, it is known that the renewal function is concave (see Brown (1980)). Conversely, Brown (1981) conjectured that DFR interrenewal times are also necessary for the concavity of the renewal function. As shown in Shanthikumar (1988), there exist counterexamples to a discrete analogue of this conjecture. Brown's conjecture in the continuous case, however, has remained open. See Szekli (1986), (1990), Hansen and Frenk (1991), Shaked and Zhu (1992), Kijima (1992), and Kebir (1997) for related results and discussions. Also relevant is the work of Lund et al. (2006), who used hazard rates and renewal sequences to study reversible Markov chains.

In this note we construct absolutely continuous distributions that do not have DFRs but nevertheless lead to concave renewal functions. That is, we give a definite answer to Brown's question in the negative. Our counterexamples have the following feature. On $\left[0, t_{1}\right]$ for some $t_{1}>0$, the interrenewal time has a DFR; on $\left[t_{1}, t_{2}\right]$ for some $t_{2}>t_{1}$, the failure rate strictly increases before decreasing again on $\left[t_{2}, \infty\right)$. It is shown that, for a suitable class of such distributions, if the increase in failure rate on $\left[t_{1}, t_{2}\right]$ is small enough, and the decrease shortly after $t_{2}$ is fast enough, then the resulting renewal density is decreasing, i.e. the renewal function is concave. In Section 2 we present the precise statements and illustrate with a numerical example. Section 3 contains the proofs.

The renewal process is closely related to compound geometric random variables. In Section 4, by adapting the arguments of de Bruijn and Erdős (1953), we give an alternative proof of Shanthikumar's (1988) result that the DFR property is closed under geometric compounding.

Received 14 September 2010; revision received 22 January 2011.

* Postal address: Department of Statistics, University of California, Irvine, CA 92697, USA.

Email address: yamingy@uci.edu 


\section{Concavity of the renewal function}

Let $F(t)$ be a distribution function on $\mathbb{R}_{+}=[0, \infty)$ with $F(0)=0$. Then the renewal function $M(t)$, i.e. the average number of renewals in $[0, t]$, for a renewal process with underlying distribution $F$ is given by

$$
M(t)=F(t)+\int_{0}^{t} M(t-x) \mathrm{d} F(x), \quad t \geq 0 .
$$

(Some authors define $M(t)+1$ as the renewal function; our results work with either definition.) If $F(t)$ is absolutely continuous with density $f(t)$ then so is $M(t)$, and a version of its density, $m(t)$, satisfies

$$
m(t)=f(t)+\int_{0}^{t} m(x) f(t-x) \mathrm{d} x, \quad t \geq 0 .
$$

A positive function $g(x), x \in \mathbb{R}_{+}$, is $\log$-convex if $\log g(x)$ is convex on $\mathbb{R}_{+}$. A distribution on $\mathbb{R}_{+}$has DFR if its survival function is log-convex on $\mathbb{R}_{+}$. We recall two fundamental results relating $M(t)$ to $F(t)$.

Theorem 1. (De Bruijn and Erdős (1953), Brown (1980), and Hansen and Frenk (1991).) The following assertions hold.

(i) If $F(t)$ has a log-convex density $f(t)$ then the renewal density $m(t)$ as in (1) is also log-convex.

(ii) If $F(t)$ is DFR then $M(t)$ is concave.

The question raised by Brown (1981) may be formulated as follows.

Conjecture 1. If the renewal function $M(t)$ is concave on $\mathbb{R}_{+}$then $F(t)$ is DFR.

Shanthikumar (1988) resolved a discrete version of this conjecture by constructing a counterexample using auxiliary results on discrete Markov chains. It has also been noted that the discrete example does not generalize and the continuous case is still open. Our main result (Proposition 1) finally disproves Conjecture 1.

Proposition 1. Let $0<t_{1}<\infty$. Let $f(t)$ be a density function that is positive on $\mathbb{R}_{+}$ and continuously differentiable on each of $I_{k}, 0 \leq k \leq 3$, where $I_{0}=\left[0, t_{1}\right], I_{1}=\left[t_{1}, t_{2}\right]$, $I_{2}=\left[t_{2}, t_{3}\right]$, and $I_{3}=\left[t_{3}, \infty\right)$, with $t_{2}, t_{3}$ to be determined. That is, $f(t)$ is continuous on $\mathbb{R}_{+}$, but $f^{\prime}(t)$ may jump at $t_{k}, k=1,2,3$. Assume that the corresponding hazard rate function $r(t)$ satisfies

(i) $r^{\prime}(t)<0, t \in I_{0}$;

(ii) on $I_{1}$ we have

$$
r(t)=\frac{\lambda}{1-\varepsilon \mathrm{e}^{\lambda t}}, \quad t_{1} \leq t \leq t_{2}
$$

for some $\varepsilon \in(0,1)$, where $\lambda>0$ is determined by $\varepsilon$ and $r\left(t_{1}\right)$;

(iii) on $I_{2}$ we have

$$
r^{\prime}(t) \leq r^{2}(t)-f(0) r(t), \quad t_{2}<t<t_{3} ;
$$

(iv) $r\left(t_{3}\right) \leq r\left(t_{1}\right)$ and $r^{\prime}(t) \leq 0, t \in I_{3}$. 

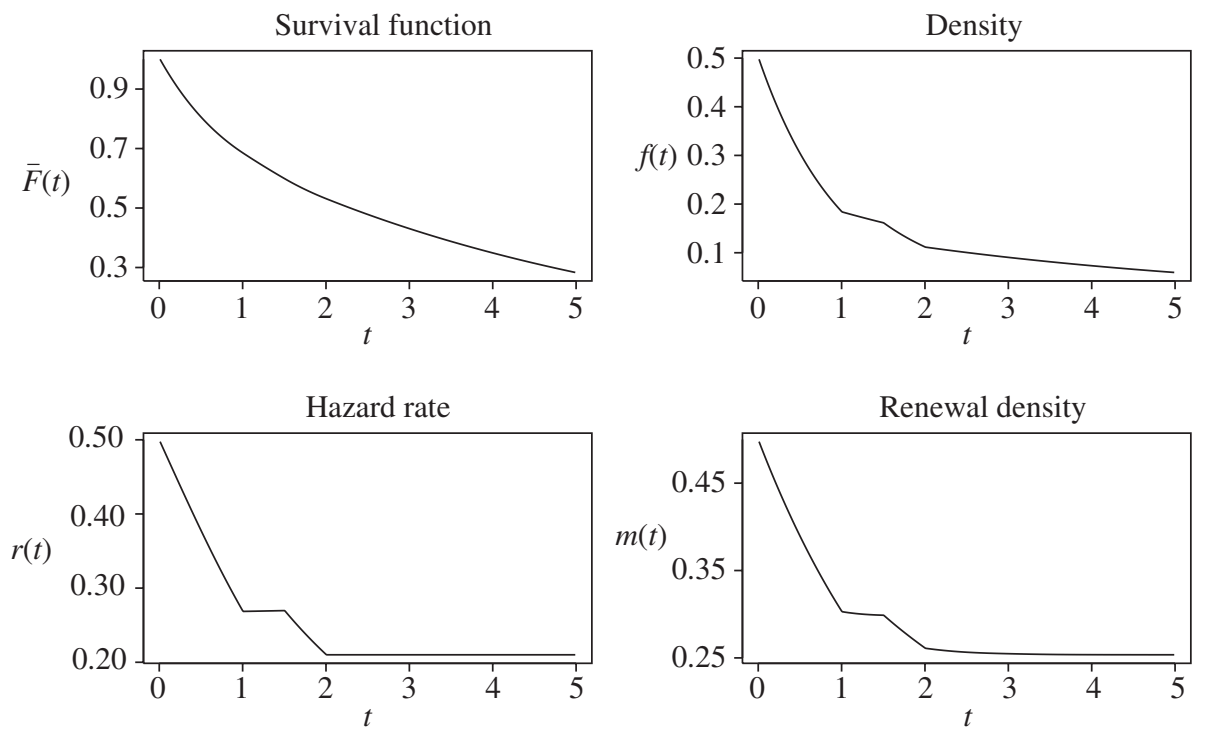

FiguRe 1: Illustration of a counterexample given by (4), (5), and (6) with $t_{1}=1, t_{2}=1.5, t_{3}=2$, and $\beta=0.02$.

Then, for small enough $\varepsilon>0$ and $t_{2}-t_{1}>0$, both depending on the specification of $r(t)$ for $t \in I_{0}$ only, the renewal density $m(t)$ given by (1) decreases on $\mathbb{R}_{+}$.

Note that $r(t)$ strictly increases on $\left[t_{1}, t_{2}\right]$. Proposition 1 therefore settles Conjecture 1 in the negative. An example of a survival function $\bar{F}$ satisfying Proposition 1(i) and (ii) is

$$
\bar{F}(t)= \begin{cases}\frac{1}{2}\left(\mathrm{e}^{-t}+1\right), & 0 \leq t \leq t_{1}, \\ \alpha \mathrm{e}^{-\lambda t}-\beta, & t_{1}<t \leq t_{2},\end{cases}
$$

where $\beta>0$, and $\alpha$ and $\lambda$ are determined by $\beta$ via

$$
\bar{F}\left(t_{1}+\right)=\bar{F}\left(t_{1}\right) \quad \text { and } \quad \bar{F}^{\prime}\left(t_{1}+\right)=\bar{F}^{\prime}\left(t_{1}-\right) .
$$

Specifically,

$$
\lambda=\left[1+(1+2 \beta) \mathrm{e}^{t_{1}}\right]^{-1}, \quad \alpha=(2 \lambda)^{-1} \mathrm{e}^{(\lambda-1) t_{1}} .
$$

The $\varepsilon$ in (2) corresponds to $\beta / \alpha$. Proposition 1(iii) says that the hazard rate should decrease fast shortly after $t_{2}$. An example based on (4) that satisfies this condition is

$$
r(t)=r\left(t_{2}\right) \mathrm{e}^{\left(t_{2}-t\right) / 2}, \quad t_{2}<t \leq t_{3},
$$

which leads to

$$
\bar{F}(t)=\bar{F}\left(t_{2}\right) \exp \left[-2 r\left(t_{2}\right)\left(1-\mathrm{e}^{\left(t_{2}-t\right) / 2}\right)\right], \quad t_{2}<t \leq t_{3} .
$$

For Proposition 1(iv), we need $t_{3} \geq t_{2}+2 \log \left(r\left(t_{2}\right) / r\left(t_{1}\right)\right)$ to ensure that $r\left(t_{3}\right) \leq r\left(t_{1}\right)$, but $r(t)$ can stay flat on $t \in I_{3}$, which gives

$$
\bar{F}(t)=\bar{F}\left(t_{3}\right) \mathrm{e}^{r\left(t_{3}\right)\left(t_{3}-t\right)}, \quad t>t_{3} .
$$

As an illustration, Figure 1 shows the survival function, density, hazard rate, and renewal density for a distribution as specified by (4), (5), and (6) with $t_{1}=1, t_{2}=1.5, t_{3}=2$, and 
$\beta=0.02$. The almost imperceptible decrease of $m(t)$ on $t \in[1,1.5]$ is verified numerically as Proposition 1 guarantees the monotonicity of $m(t)$ for small enough $\beta>0$ and $t_{2}-t_{1}>0$ but does not specify how small $\beta$ or $t_{2}-t_{1}$ has to be.

\section{Proof of Proposition 1}

We first establish a simple but useful identity.

Lemma 1. Let $r(t)$ and $\bar{F}(t)$ denote the hazard rate and survival functions, respectively, for a distribution with density $f(t)$ on $\mathbb{R}_{+}$. Assume that $f(t)$ is absolutely continuous and that $f^{\prime}(t)$ is bounded on every compact subinterval of $\mathbb{R}_{+}$. Then the renewal density $m(t)$ as defined by (1) satisfies

$$
m^{\prime}(t)=r^{\prime}(t) \bar{F}(t)+\int_{0}^{t} m^{\prime}(x)[r(t-x)-r(t)] \bar{F}(t-x) \mathrm{d} x, \quad t>0 .
$$

A discrete version of (7) can be traced back to Kaluza (1928).

Proof of Lemma 1. The conditions guarantee that $m(t)$ is absolutely continuous. In fact, we may differentiate under the integral sign in (1) and obtain

$$
m^{\prime}(t)=f^{\prime}(t)+m(t) f(0)+\int_{0}^{t} m(x) f^{\prime}(t-x) \mathrm{d} x, \quad t>0 .
$$

Integration by parts then yields

$$
m^{\prime}(t)=f^{\prime}(t)+m(0) f(t)+\int_{0}^{t} m^{\prime}(x) f(t-x) \mathrm{d} x, \quad t>0 .
$$

We also have

$$
\begin{aligned}
\int_{0}^{t} m^{\prime}(x) \bar{F}(t-x) \mathrm{d} x & =m(t)-f(0) \bar{F}(t)-\int_{0}^{t} m(x) f(t-x) \mathrm{d} x \\
& =f(t)-f(0) \bar{F}(t),
\end{aligned}
$$

where we have used integration by parts in the first step and (1) in the second step. The identity (7) follows by expanding its right-hand side and applying (9) and (10) to simplify.

Proof of Proposition 1. Since $f^{\prime}(t)$ is piecewise continuous, so is $m^{\prime}(t)$, as seen from (8). We have $m^{\prime}(0+)=r^{\prime}(0+)<0$. Suppose that $m^{\prime}(t)$ becomes nonnegative on $I_{0}$. Then letting $t_{*}$ be the smallest $t \in\left(0, t_{1}\right)$ such that $m^{\prime}(t) \geq 0$ we have $m^{\prime}(x)<0,0<x<t_{*}$, and, by Proposition 1(i), $r^{\prime}\left(t_{*}\right)<0, r\left(t_{*}-x\right)-r\left(t_{*}\right)>0,0<x<t_{*}$. It follows from (7) that $m^{\prime}\left(t_{*}\right)<0$, a contradiction. Thus, $m^{\prime}(t)<0, t \in I_{0}$. In fact, applying (7) again yields

$$
m^{\prime}(t)<r^{\prime}(t) \bar{F}(t), \quad t \in I_{0},
$$

where the left derivatives are used if $t=t_{1}$.

By (2) we have

$$
r^{\prime}(t)=\left(\lambda^{-1} r(t)-1\right) r(t), \quad t_{1}<t<t_{2},
$$

where $\lambda$ is determined from $\varepsilon$ via $r\left(t_{1}\right)=\lambda /\left(1-\varepsilon \mathrm{e}^{\lambda t_{1}}\right)$. For fixed $r\left(t_{1}\right)$ as $\varepsilon \downarrow 0$, we have $\lambda \uparrow r\left(t_{1}\right)$ and, hence, $r^{\prime}\left(t_{1}+\right) \downarrow 0$. Defining $\delta=m^{\prime}\left(t_{1}-\right)-r^{\prime}\left(t_{1}-\right) \bar{F}\left(t_{1}\right)$, and noting that $\delta<0$ by (11), we obtain

$$
m^{\prime}\left(t_{1}+\right)=r^{\prime}\left(t_{1}+\right) \bar{F}\left(t_{1}\right)+\delta<0 \text { for small enough } \varepsilon>0 .
$$


Because $m^{\prime}(t)$ is continuous on $\left(t_{1}, t_{2}\right)$, and $m^{\prime}\left(t_{1}+\right)<0$, we have $m^{\prime}(t)<0, t \in\left(t_{1}, t_{2}\right)$, if $t_{2}-t_{1}$ is small enough. Thus, $m(t)$ decreases on $I_{1}$.

Also, $m(t)$ must strictly decrease on $I_{2}$. Assume the contrary, and let $t^{*}$ be the smallest $t \in\left[t_{2}, t_{3}\right)$ such that $m^{\prime}(t+) \geq 0$. Then (9) gives

$$
0 \leq m^{\prime}\left(t^{*}+\right)<f^{\prime}\left(t^{*}+\right)+m(0) f\left(t^{*}\right),
$$

because inside the integral $m^{\prime}(x)<0, x \in\left[0, t^{*}\right)$. However, by (3) we have

$$
f^{\prime}\left(t^{*}+\right)+m(0) f\left(t^{*}\right)=\bar{F}\left(t^{*}\right)\left[r^{\prime}\left(t^{*}+\right)-r^{2}\left(t^{*}\right)+f(0) r\left(t^{*}\right)\right] \leq 0,
$$

which contradicts (12).

Finally, we show that $m(t)$ decreases on $I_{3}$ by applying (7) again. The assumptions $r\left(t_{3}\right) \leq$ $r\left(t_{1}\right)$ and $r^{\prime}(t) \leq 0, t \in I_{3}$, ensure that $r(t-x) \geq r(t), 0<x<t, t>t_{3}$, with strict inequality if $t-x<t_{1}$. It is already shown that $m^{\prime}(x)<0$ for $x<t_{3}$. Thus, (7) implies that $m^{\prime}\left(t_{3}+\right)<0$. The same argument proving that $m^{\prime}(t)<0$ for $t \in I_{0}$ then shows that $m(t)$ decreases on $I_{3}$.

\section{Preservation of DFR under geometric compounding}

Compound geometric random variables appear naturally in areas such as queueing theory (see, e.g. Szekli (1986)) and financial risk modeling. It is well known that log-convexity is closed under geometric compounding (this is essentially Theorem 1(i)). Shanthikumar (1988) showed that the DFR property is also closed under geometric compounding. This was achieved by establishing auxiliary results on discrete Markov chains. It may be worthwhile to note that the argument of de Bruijn and Erdős (1953) can be adapted to give a short proof of Shanthikumar's (1988) result (Theorem 2(i) below). The same argument yields a parallel result (Theorem 2(ii)) concerning the increasing failure rate (IFR) property.

Theorem 2. Let $X$ be a random variable on $\mathbb{N}=\{1,2, \ldots\}$, and let $T$ be geometric with parameter $p \in(0,1)$, i.e. $\operatorname{Pr}(T=n)=p q^{n-1}, n=1,2, \ldots, q \equiv 1-p$. Define the random sum $Y \equiv \sum_{k=1}^{T} X_{k}$, where $X_{1}, X_{2}, \ldots$ are independent and identically distributed with generic copy $X$ and also independent of $T$.

(i) If $\log \operatorname{Pr}(X \geq n)$ is convex in $n \in \mathbb{N}$, i.e. $X$ is discrete DFR, then so is $Y$.

(ii) If $\log \operatorname{Pr}(Y \geq n)$ is concave in $n \in \mathbb{N}$, i.e. $Y$ is discrete IFR, then so is $X$.

Proof. Define

$$
f_{n}=\operatorname{Pr}(X=n), \quad \bar{F}_{n}=\operatorname{Pr}(X \geq n), \quad g_{n}=\operatorname{Pr}(Y=n), \quad \bar{G}_{n}=\operatorname{Pr}(Y \geq n) .
$$

We have the recursions

$$
g_{n}=p f_{n}+q \sum_{k=1}^{n-1} f_{k} g_{n-k}, \quad \bar{G}_{n}=\bar{F}_{n}+q \sum_{k=1}^{n-1} f_{k} \bar{G}_{n-k}, \quad n=1,2, \ldots
$$

The following identity is analogous to Equation (7) of de Bruijn and Erdős (1953); Hansen (1988) used similar identities for compound Poisson probabilities (see Yu (2009) for related 
work). It is proved by expanding the right-hand side and then applying (13):

$$
\begin{aligned}
\bar{F}_{n}\left(\bar{G}_{n+2} \bar{G}_{n}-\bar{G}_{n+1}^{2}\right)= & p \bar{G}_{n}\left(\bar{F}_{n+2} \bar{F}_{n}-\bar{F}_{n+1}^{2}\right) \\
& +q \sum_{k=2}^{n}\left(\bar{F}_{n+1} f_{k-1}-\bar{F}_{n} f_{k}\right)\left(\bar{G}_{n+1} \bar{G}_{n+1-k}-\bar{G}_{n} \bar{G}_{n+2-k}\right) .
\end{aligned}
$$

In particular, $\bar{G}_{3} \bar{G}_{1}-\bar{G}_{2}^{2}=p\left(\bar{F}_{3} \bar{F}_{1}-\bar{F}_{2}^{2}\right)$. Assuming that $\bar{F}_{n}$ is log-convex, we obtain $\bar{G}_{n+1} \bar{G}_{n+1-k} \geq \bar{G}_{n} \bar{G}_{n+2-k}, 2 \leq k \leq n$, and $\bar{G}_{n+2} \bar{G}_{n} \geq \bar{G}_{n+1}^{2}, n \geq 1$, by induction from (14). Thus, $\bar{G}_{n}$ is log-convex in $n \in \mathbb{N}$, i.e. $Y$ is discrete DFR, and part (i) is proved. Similarly, assuming that $\bar{G}_{n}$ is log-concave, we obtain $\bar{F}_{n+1} f_{k-1} \leq \bar{F}_{n} f_{k}, 2 \leq k \leq n$, and $\bar{F}_{n+2} \bar{F}_{n} \leq$ $\bar{F}_{n+1}^{2}, n \geq 1$, by induction. Thus, $\bar{F}_{n}$ is log-concave in $n \in \mathbb{N}$, i.e. $X$ is discrete IFR, and part (ii) is proved.

\section{Acknowledgements}

The author would like to thank Mark Brown, George Shanthikumar, and Ryszard Szekli for their helpful comments.

\section{References}

Brown, M. (1980). Bounds, inequalities, and monotonicity properties for some specialized renewal processes. Ann. Prob. 8, 227-240.

Brown, M. (1981). Further monotonicity properties for specialized renewal processes. Ann. Prob. 9, 891-895.

De Bruijn, N. G. AND ERdős, P. (1953). On a recursion formula and some Tauberian theorems. J. Res. Nat. Bur. Standards 50, 161-164.

Hansen, B. G. (1988). On log-concave and log-convex infinitely divisible sequences and densities. Ann. Prob. 16, 1832-1839.

Hansen, B. G. And Frenk, J. B. G. (1991). Some monotonicity properties of the delayed renewal function. J. Appl. Prob. 28, 811-821.

KaluZA, T. (1928). Über die Koeffizienten reziproker Potenzreihen. Math. Z. 28, 161-170.

KeBIR, Y. (1997). Laplace transforms and the renewal equation. J. Appl. Prob. 34, 395-403.

KiJima, M. (1992). Further monotonicity properties of renewal processes. Adv. Appl. Prob. 24, 575-588.

Lund, R., Zhao, Y. And Kiessler, P. C. (2006). A monotonicity in reversible Markov chains. J. Appl. Prob. 43, 486-499.

Shaked, M. And Zhu, H. (1992). Some results on block replacement policies and renewal theory. J. Appl. Prob. 29, 932-946.

ShANTHIKUMAR, J. G. (1988). DFR property of first-passage times and its preservation under geometric compounding. Ann. Prob. 16, 397-406.

SzekLI, R. (1986). On the concavity of the waiting-time distribution in some GI/G/1 queues. J. Appl. Prob. 23, $555-561$.

SzEKLI, R. (1990). On the concavity of the infinitesimal renewal function. Statist. Prob. Lett. 10, 181-184.

Yu, Y. (2009). On the entropy of compound distributions on nonnegative integers. IEEE Trans. Inf. Theory 55, 36453650 . 